\title{
NITROGEN FIXING BACTERIA OF SPRING WHEAT ROOT ZONE
}

\author{
Nadkernychna O. V., Kopylov E. P.
}

Institute of Agricultural microbiology and agricultural production NAAS 97, Shevchenko str., Chernihiv, 14027, Ukraine

The paper presents the study of active nitrogen fixation bacteria of genera Azotobacter, Azospirillum, Bacillus, Flavobacterium, Enterobacter and Pseudomonas isolated from root zone of spring wheat plants. The ability of selected diazotrophs to form associative systems with spring wheat was investigated. The most significant increase of molecular nitrogen fixation activity in root zone of plants was observed under the Azospirillum species background.

Keywords: diazotrophs, spring wheat, associative nitrogen fixation.

The problem of biological fixation of atmospheric nitrogen, despite almost a century history, remains one of the major in biological science. In the 70s of the last century the phenomenon of increased nitrogen fixation activity in the root zone of plants, called associative nitrogen fixation was discovered. Studies in this area began with the works of J. Dobereiner [1], where she suggested that the growth and biomass formation of tropical grasses take place owing to atmospheric nitrogen fixation. Many subsequent studies have shown that nitrogen-fixing bacteria easily form associations with various non-legume crops where donor plant provides diazotrophs with available sources of carbon and phosphorus and creates favorable conditions for the nitrogen fixation, and uses, in turn, assimilated nitrogen. Due to the ability to form such associations, diazotrophs adapt better to the environment and have advantages in nutrients supply over other microrganisms.

The ability to establish stable associations of cultivated plants with diazotrophs, analytically selected from agrobiocenoses, is most catching in practical terms and can be considered as the most important measure aimed on the conservation of biological diversity and environmental sustainability.

The aim of this study was to investigate the microbial nitrogen fixation communities of root zone of spring wheat plants and to obtain the culture of diazotrophs that can form active associations with plants.

Materials and methods. Nitrogen fixating bacteria isolated from the inter-row soil, soil washed from rhizosphere and roots of spring wheat plants grown under the small-pot field experiments on shallow black leached light loess loamy soils.

Active diazotrophs were isolated from batch culture after definition of their nitrogenase activity [2]. Batch cultures, which nitrogenase activity was greater than $0.3 \mathrm{mg}$ of nitrogen per $1 \mathrm{ml}$ of culture medium, were cultivated on solid culture media: potato agar, Kaseras, Dobereyner, Eshbi, and agar medium with glycerol.

Nitrogenase activity of pure diazotrophs cultures was detected with acetylene method on gas chromatograph "Chrom-4" with flame-ionization detector. Column length $-370 \mathrm{~cm}$ filled with chromosorb with $\beta$ - $\beta$-oxydipropionitrile. Thermostat temperature $-50{ }^{\circ} \mathrm{C}$, carrier gas - nitrogen, gas flow (in $\mathrm{ml} /$ minute): hydrogen -30 , nitrogen - 100, air - 500 . 
Green house experiments were conducted in black leached soils with following agrochemical parameters: humus content in the plow layer $-3.6 \%$, mobile forms of phosphorus (by Kirsanov) - 210-240 $\mathrm{mg}_{2} \mathrm{P}_{5}$, exchangeable potassium (by Kirsanov) $-160-170 \mathrm{mg}$ of $\mathrm{K}_{2} \mathrm{O}$ per $1 \mathrm{~kg}$ of soil $\mathrm{pHw}-6.5$. Soil moisture was maintained at $60 \%$ of full water capacity. Used pots $-12 \times 15 \mathrm{~cm}$, capacity -2.01 . Seeds of spring wheat of Rannya 93 variety were sown to a depth of $2.0 \mathrm{~cm}, 20$ plants per pot. Experiment repetition - 10-fold.

Scheme of experiments: $1-$ w/o inoculation (control), 2 -inoculation with diazotrophs. Bacterial cultures were grown for three days on the following medium: Pseudomonas - solid medium with glycerol, Azotobacter - Eshbi medium with mannitol, Flavobacterium - Eshbi medium with glucose, Bacillus - potato agar with glucose, Enterobacter - pea agar medium, Azospirillum -potato agar with malate. Presowing seeds treatment was performed with the suspension of diazotrophs with the rate of 200-300 thousand bacterial cells per seed. Experiments Duration - 30-40 days after emergence. At the end of this period nitrogen fixation activity was determined by acetylene method in undisturbed soil monolith with roots.

Results and discussion. The results obtained have shown favorable conditions for the development of nitrogen fixing bacteria of different taxonomic and ecologicaltrophic groups in rhizosphere and rhizoplan of spring wheat plants.

More than 1000 diazotroph cultures were analytically selected from different areas of root zone of spring wheat. It was established that only 310 batch cultures (31\%) have displayed nitrogenase activity over $0.3 \mathrm{mg}$ of nitrogen per $1 \mathrm{ml}$ of culture medium per day. 60 cultures with high nitrogenase activity were isolated into the pure culture.

Generic distribution of the selected active diazotroph strains is shown on Figure 1.

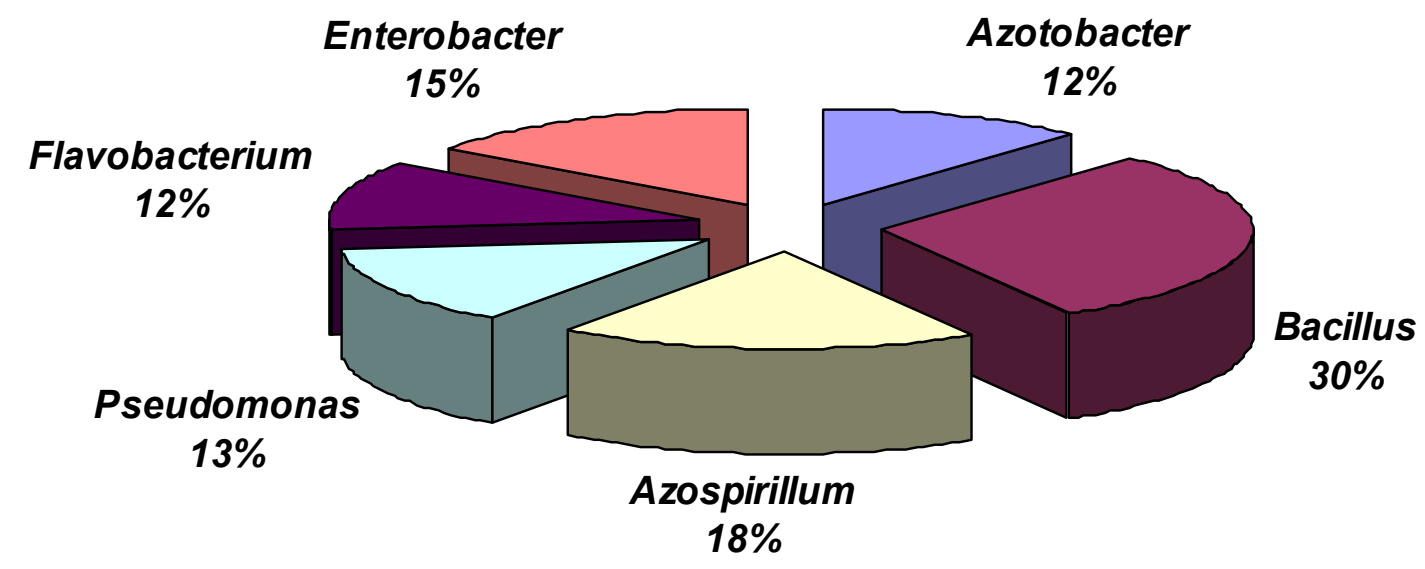

FIGURE. 1. Generic composition of nitrogen fixing associations of root zone of spring wheat plants.

Most active strains (30\%) belonged to the Bacillus genus. A number of other families have not differ significantly: 18\% - bacteria of Azospirillum genus, 15\% Enterobacter, 13\% - Pseudomonas and 12\% - Flavobacterium and Azotobacter. 
Ability of the selected active diazotroph strains to form effective associations with spring wheat plants was evaluated in green house experiments. As is known from the literature diazotrophic bacteria can not be referred as associative microorganisms based only on their ability to actively fix molecular nitrogen in pure culture in a nutrient medium. It is necessary to ensure its ability to enhance associative nitrogen fixation at introduction into the root zone of plants, since there is no direct relationship between nitrogenase activity in pure culture and in association with plants. Some strains that were able to actively fix nitrogen in pure culture were unable to form effective nitrogen fixing associations with plants and vice versa [3]. This is consistent with some authors stating that the controlling factor in the formation of effective diazotrophic associations with plants and increase of inoculated plants productivity is multifunctional effect of rhizospheric diazotrophs on plants [4], and as a result, improvement of nitrogen nutrition of crops due to the fixation of molecular nitrogen.

Figure 2 shows the ability of diazotrophs of Azotobacter genus to form associations with spring wheat plants and enhance nitrogenase activity in the root zone.

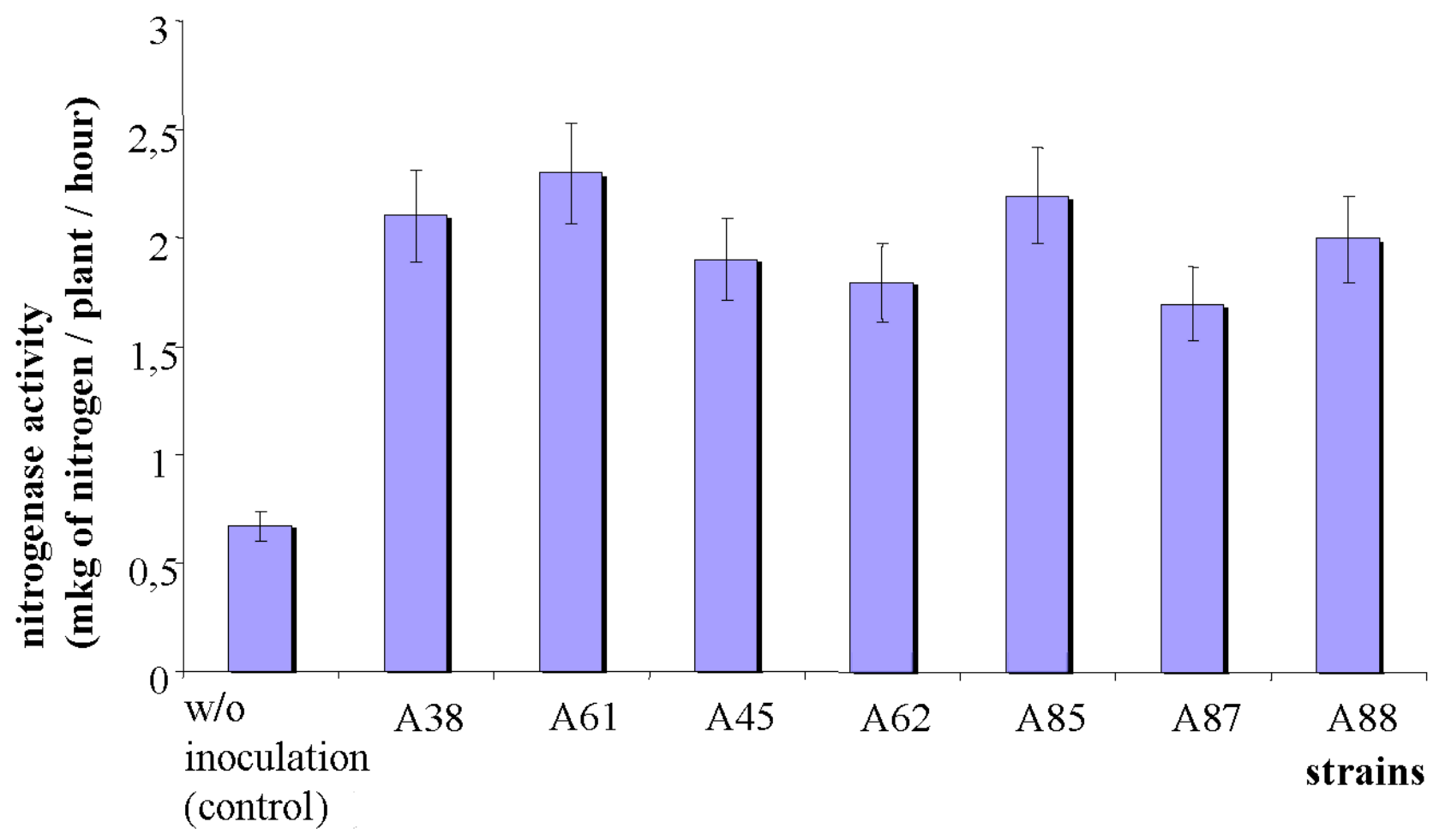

FIGURE. 2. Nitrogenase activity of Azotobacter strains in association with spring wheat plants of Rannya 93 variety.

Data depicted on Figure 2, shows associative nitrogen fixation activity in the root zone of spring wheat plants inoculated with Azotobacter, ranged from 1.7 to 2.3 mg of nitrogen / plant / hour depending on the strain activity. All isolates have promoted increase of this parameter in $2.8-3.8$ times as comparing to the variant without inoculation. It was noted that pure Azotobacter cultures have significantly differed from each other in nitrogen fixing activity (in $1.5-4.3$ times), while the 
difference in associative nitrogen fixation values at inoculation of spring wheat of Rannya 93 variety with these strains was significantly lower - not exceeding $35 \%$.

Ability of Bacillus genera diazotrophs to form associations with spring wheat and enhance nitrogen-fixing activity in plants root zone is shown on Figure 3.

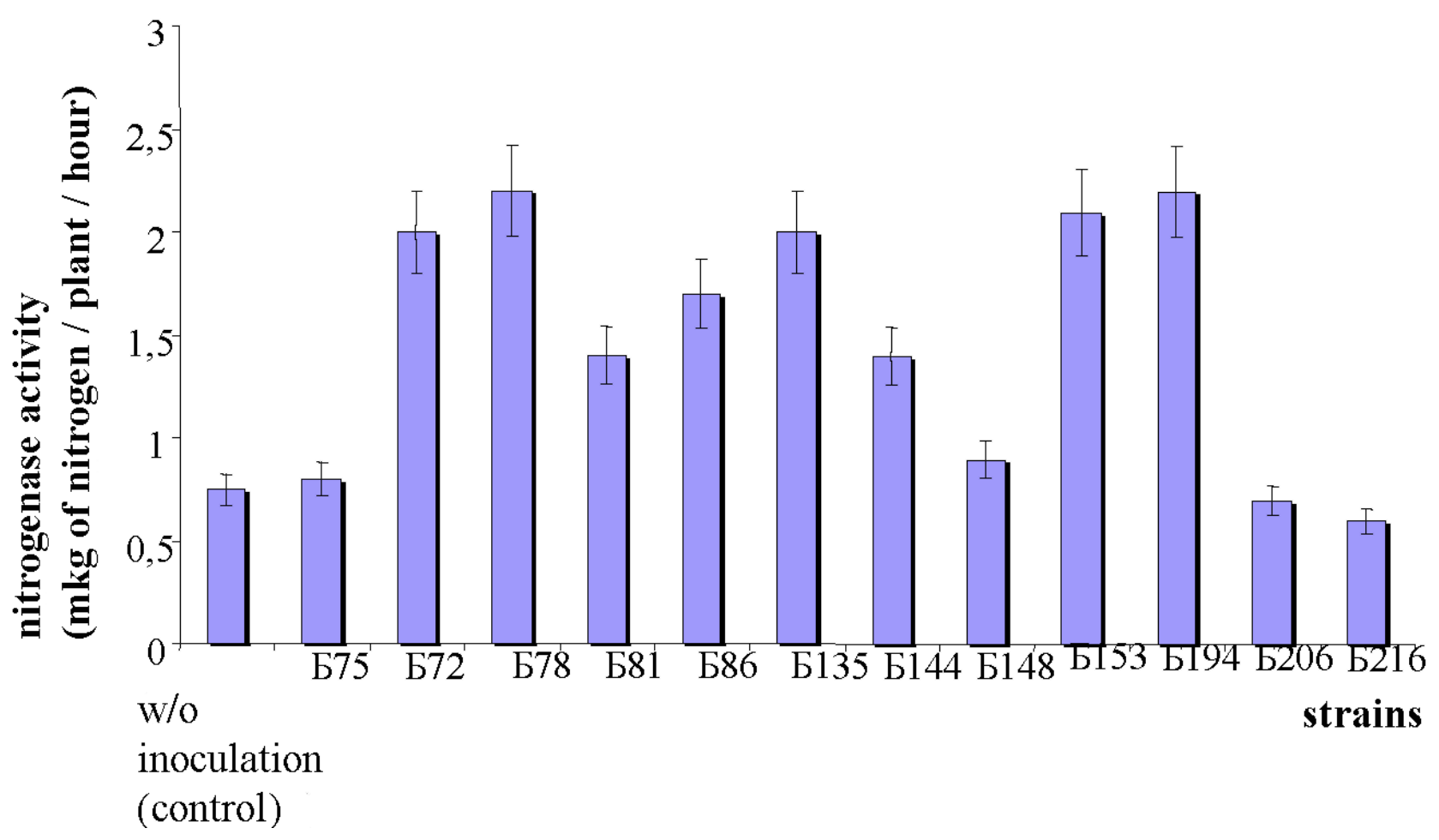

FIGURE. 3. Nitrogenase activity of Bacillus strains in association with spring wheat plants of Rannya 93 variety.

Data presented on Figure 3 shows that only few isolated bacilli strains, capable of fixing molecular nitrogen in pure culture, were able to form active nitrogen fixing association with spring wheat plants. Thus, nitrogenase activity in the root zone of plants inoculated with Bacillus sp. B75, Bacillus sp. B148, Bacillus sp. B206, was at the control plants level. The remaining strains have increased associative nitrogen fixation activity, ranged from 1.4 to $2.3 \mathrm{mg}$ of nitrogen / plant / hour. However, no correlation between the activity of nitrogen fixation in pure culture and in association with spring wheat plants was observed. Bacillus sp. B75 strain has showed maximum nitrogenase activity in pure culture $(2.8 \mathrm{mg}$ of nitrogen / $1 \mathrm{ml}$ of culture medium / day), and at the same time, its introduction into the root zone of plants have not influenced on associative nitrogen fixation. Some bacilli strains (Bacillus sp. B78, Bacillus sp. B135, Bacillus sp. B194) possess high nitrogenase activity both in pure culture and in association with of spring wheat plants.

Selected diazotrophs of Pseudomonas genus were able to form active associations with spring wheat (Fig. 4). 


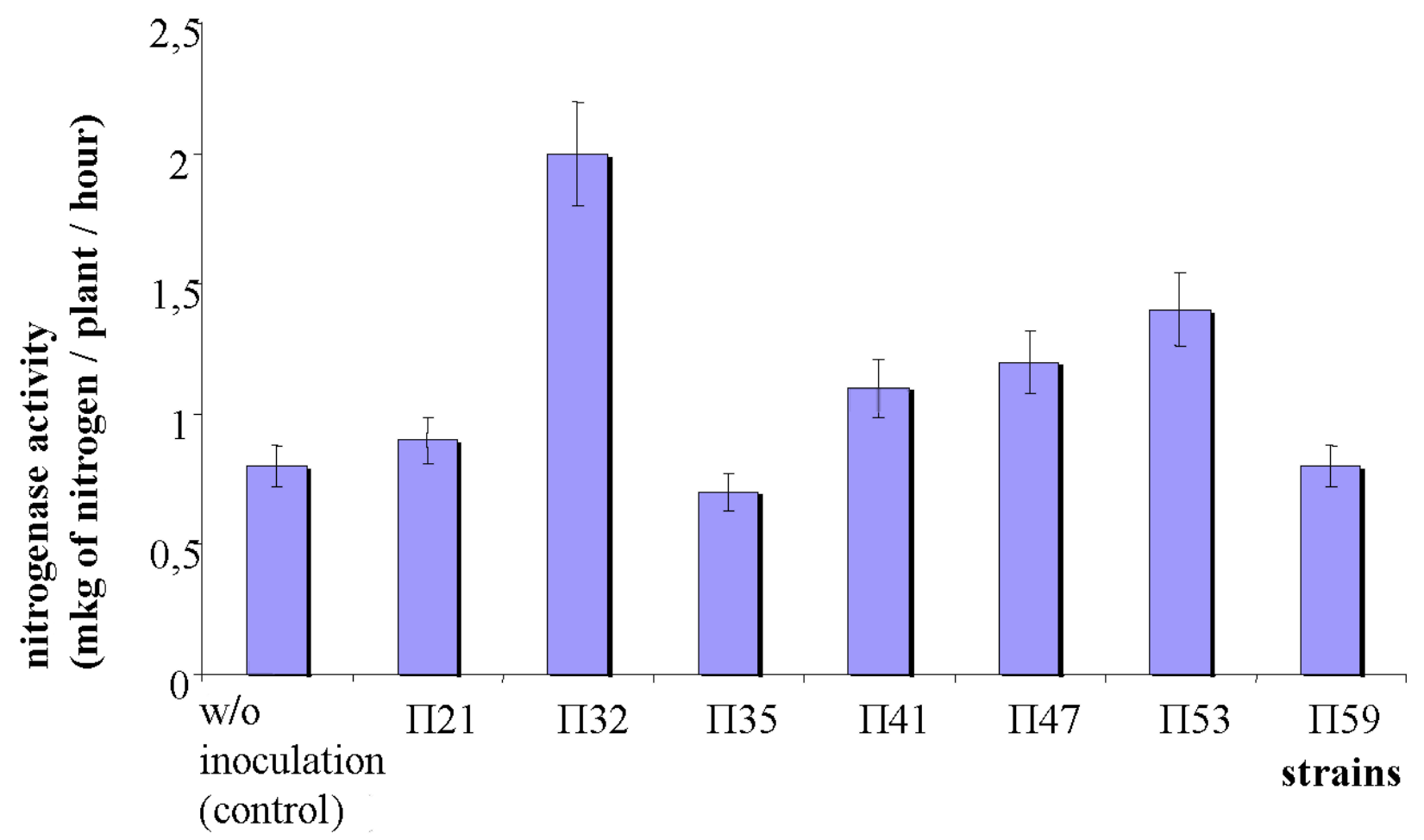

FIGURE. 4. Nitrogenase activity of Pseudomonas strains in association with spring wheat plants of Rannya 93 variety.

Results obtained have shown that only four strains of Pseudomonas genus, (Pseudomonas sp P32, Pseudomonas sp P41, Pseudomonas sp Pseudomonas sp P47 and P53) have promoted molecular nitrogen fixation in the root zone of spring wheat plants through the formation of active associations with host plants. Nnitrogenase activity of Pseudomonas - spring wheat systems was on the same level as the associative systems of Azotobacter with spring wheat and spring wheat - Bacillus sp.

Diazotrophs of Flavobacterium genus were unable to enhance nitrogen fixation activity in the plants root zone, i.e., these strains were not able to form active associative systems with the spring wheat (Fig. 5).

Ability of diazotrophs of Enterobacter genus to form associations with spring wheat and enhance nitrogenase activity in the root zone of plants is shown on Fig. 6.

According to the data obtained, out of 9 strains of Enterobacteriaceae, characterized with high nitrogen fixation activity of in pure culture (from 2.2 to 4.7 $\mathrm{mg}$ of nitrogen / $1 \mathrm{ml}$ of culture medium / day) only 3 strains (Enterobacter sp. E18, Enterobacter sp . E80 and Enterobacter sp. E388) were not able to increase nitrogenase activity in the root zone of spring wheat plants. The remaining strains were able to form associative system of Enterobacteriaceae with spring wheat, although the activity of this system was low (from 0.9 to $1.4 \mathrm{mg}$ nitrogen / plant / hour) and was lower as comparing to the associative systems of Azotobacter or Bacillus with spring wheat.

Study of ability of Azospirillum bacteria to form associative systems with spring wheat have showed the largest nitrogen fixing activity of these bacteria as compared to others investigated microorganisms. Their introduction into the root system of plants ensures formation of active associations of Azospirillum strains with spring wheat (Fig. 7). 


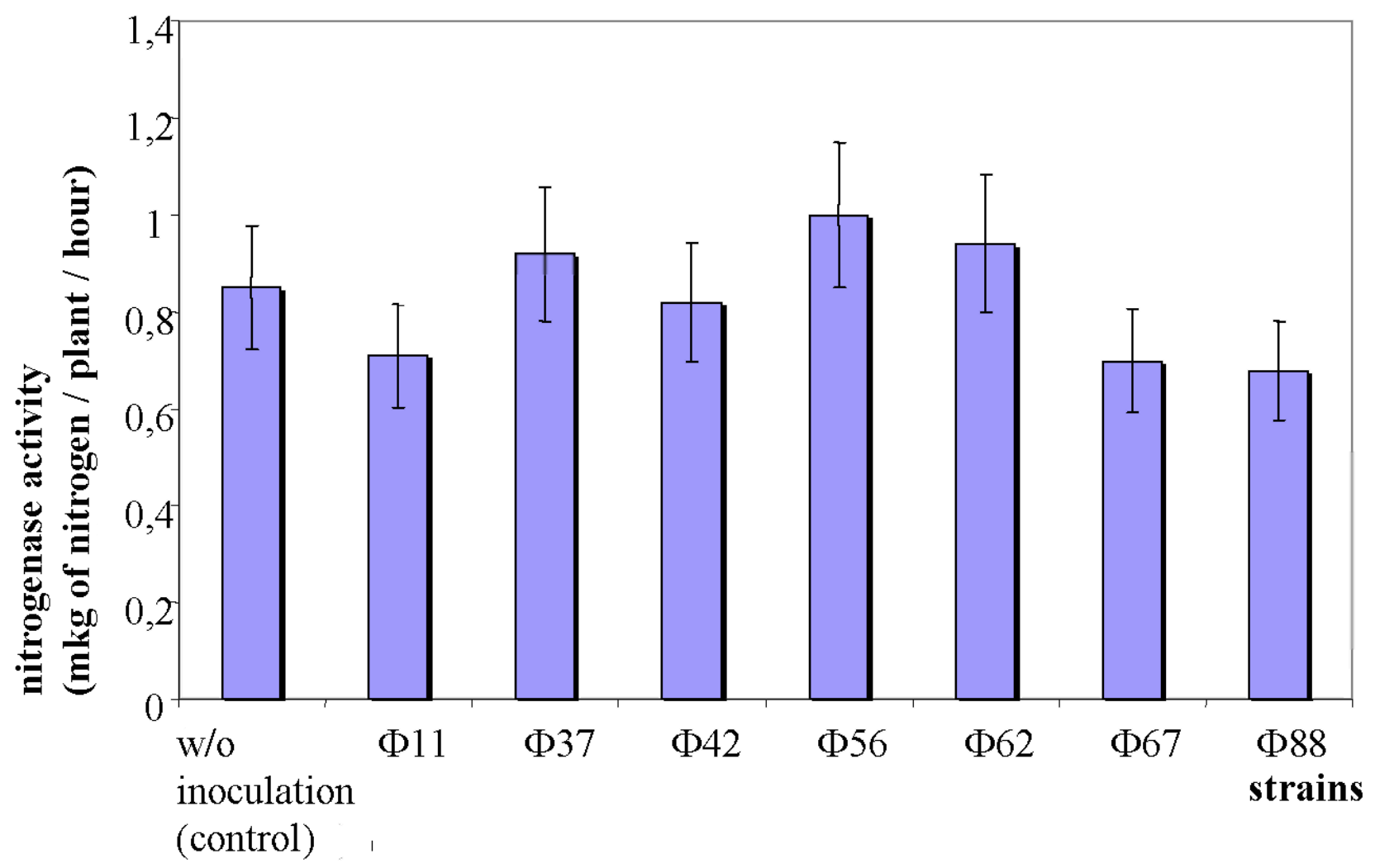

FIGURE. 5. Nitrogenase activity of Flavobacterium strains in association with spring wheat plants of Rannya 93 variety.

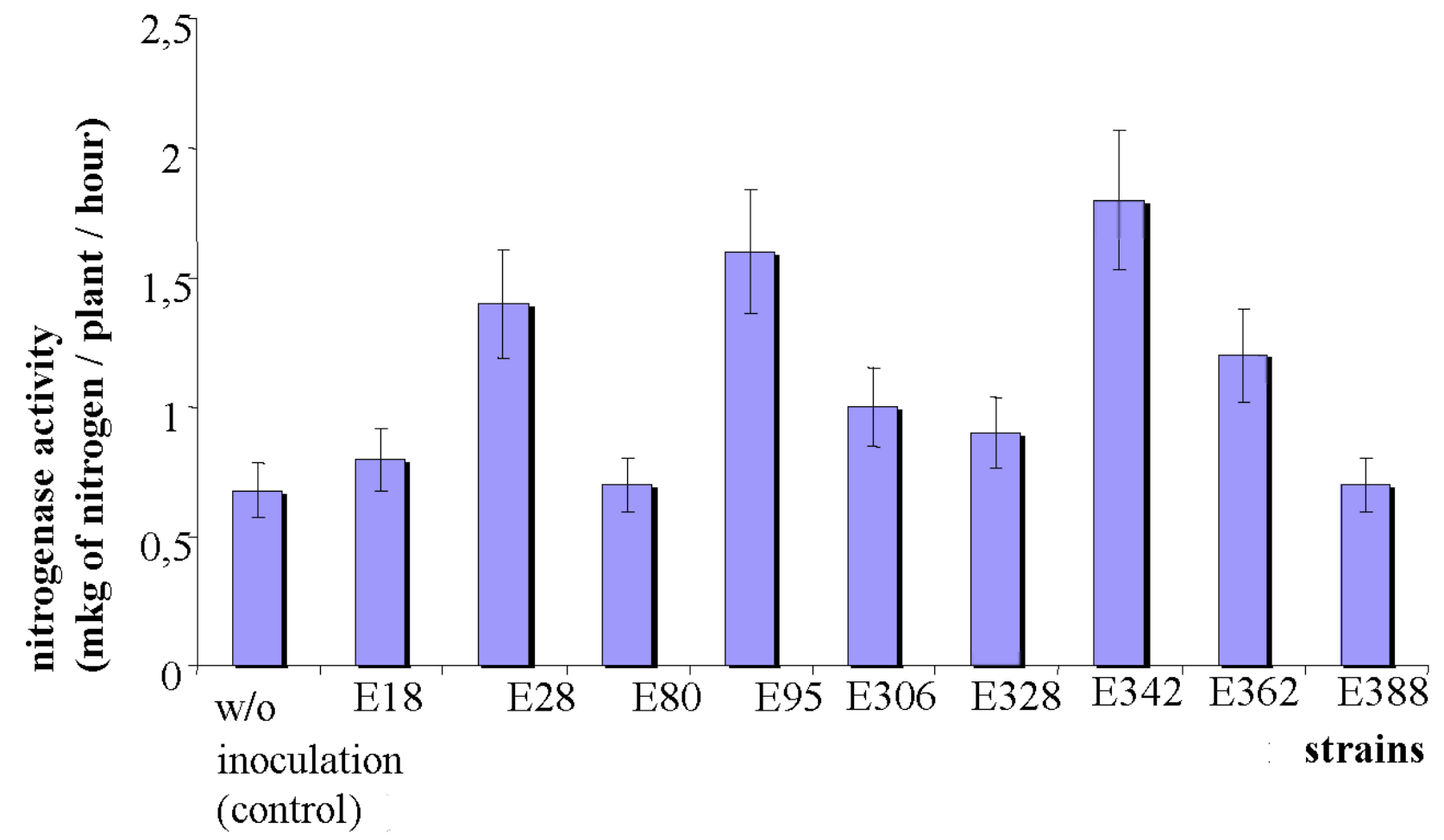

FIGURE. 6. Nitrogenase activity of Enterobacteriaceae family strains in association with spring wheat plants of Rannya 93 variety. 


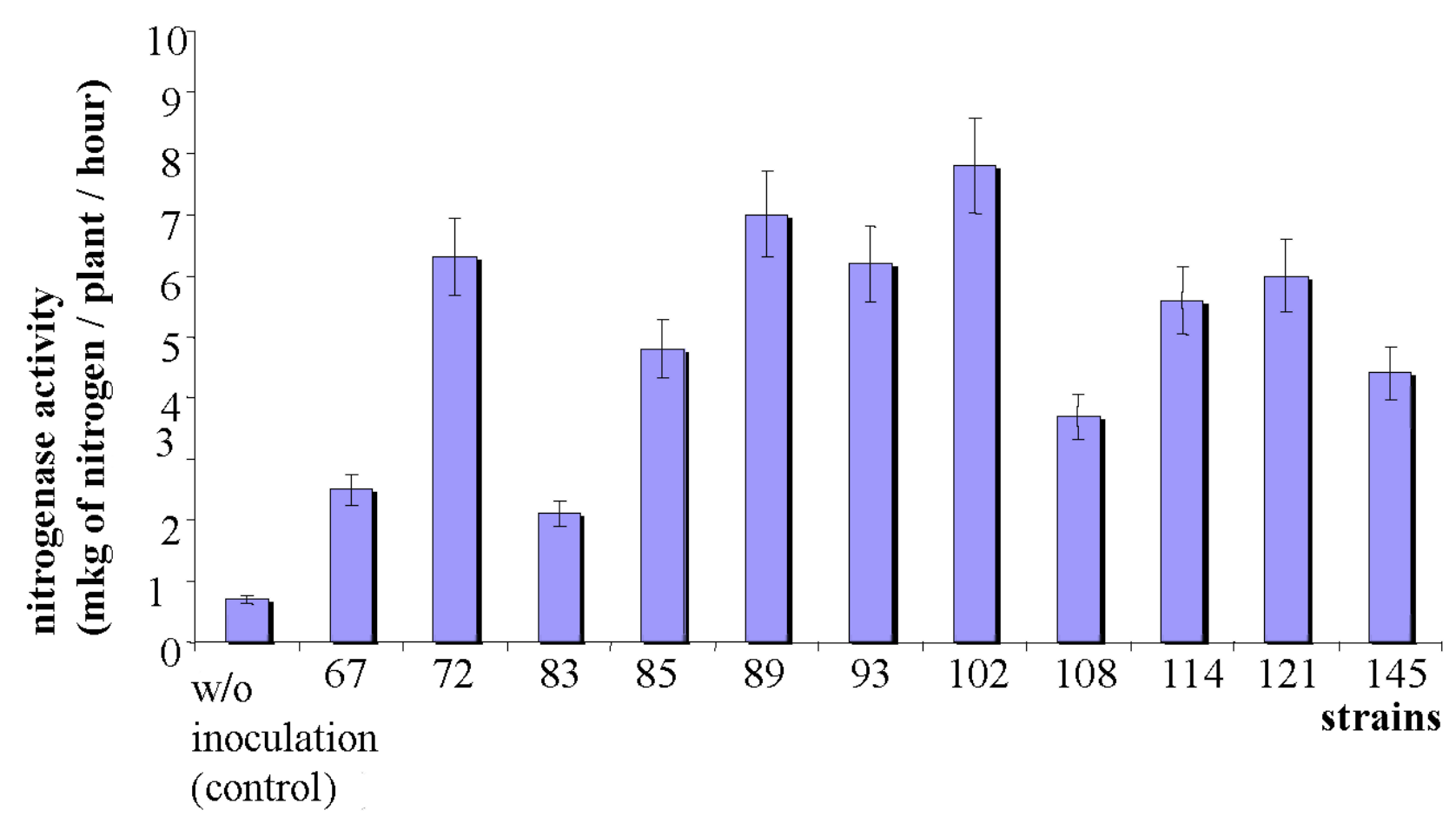

FIGURE. 7. Nitrogenase activity of Azospirillum strains in association with spring wheat plants of Rannya 93 variety.

Data on Figure 7 demonstrates that all isolates of Azospirillum genera are able to form associations with spring wheat plants possessing high nitrogenase activity ranging from 2.1 to $7.8 \mathrm{mg}$ of nitrogen / plant / hour. However, as it was shown for other diazotrophs, no direct relationship between the activity of molecular nitrogen fixation in pure culture and in associations with plants was observed. Thus, Azospirillum sp. 83 strain, characterized with high nitrogenase activity - $10.4 \mathrm{mg}$ of nitrogen / $1 \mathrm{ml}$ of culture medium / day in pure culture, in association with spring wheat plants have showed the lowest activity $-2.1 \mathrm{mg}$ nitrogen / plant / hour. At the same time, Azospirillum sp. 102 strain have showed the highest nitrogenase activity in pure culture and in association with plants.

It was shown that diazotrophs of Azotobacter, Azospirillum, Bacillus, Enterobacterand Pseudomonas genera isolated from root zone of spring wheat can create associative systems with host plants, with the Azospirillum strains forming the most active nitrogen fixing associations with spring wheat.

To the point, the studies of diazotrophs of spring wheat have shown that the microbial - plants root communities preserve an inexhaustible genetic potential of diazotrophs and, using analytical selection techniques, it is possible to obtain strains with the potential efficiency that will significantly prevail over the existing biological agents used in agricultural microbial preparations.

Analyze of literature data together with the results of studies of nitrogen fixing associations in root zone of spring wheat plants, indicate that the structure and function of diazotrophs complex in crop root zone (in our case - cereals) has its own characteristics, but at the same time, some groups of diazotrophs inherent in all investigated grain crops. Thus, nitrogen fixing bacteria of Azotobacter, Azospirillum, Bacillus, Enterobacter and Pseudomonas genera that actively interact with plants are 
typical not only for the root zone of spring wheat plants, but also for spring barley and triticale as well as for winter wheat and rye $[3,5,6,7]$.

Nevertheless, some interaction features of diazotrophic bacteria with grain crops in the micro-and makroorganisms system can be illustrated on the example of Enterobacter and agrobacteria.

Ukraine has a number of bacterial preparations, having associative diazotrophs as bio-agents. One of these preparations is Rhizoenteryn, based on the strain of Enterobacter aerogenes 30F. This strain, isolated from a meadow-black soil rhizosphere of rice (Krasnodar Territory) was originally used to enhance the yield of rice [8], but appear to be more effective under spring barley. Field experiments conducted in many regions of Ukraine, have shown that use of Enterobacter can increase barley yield, and significantly increase nitrogenase activity in the rhizosphere of inoculated plants [6].

Study of the E. aerogenes 30F strain ability to acclimatize in the root zone of spring barley plants showed that the bacteria colonize the rhizosphere soil and are especially active in plants rhizoplan, ensuring success of Enterobacter inoculation [5]. In green house experiments with spring wheat it was also shown the effectiveness of plants inoculation with active Enterobacter strains and the ability of bacteria of this genus to form active associations with the this crop.

At the same time, studies of winter grain crops indicate the inefficiency of Enterobacter use. Thus, despite the relatively high nitrogenase activity in the pure culture, bacteria of Enterobacter genus have not promoted activity of nitrogen fixation in winter rye, specifically, they were not able to form an active associative system with this crop and were ineffective on this culture [3]. Attempts to apply Rhizoenteryn on winter wheat have showed slight increase of the molecular nitrogen fixation activity in the rhizosphere of inoculated plants, but grain yield and protein content have remained at the control levels [6].

A similar pattern was observed with the perspective for several crops associative diazotroph - Rhizobium radiobacter.

It is known, that some Rhizobium strains are able to actively fix molecular nitrogen in pure culture and in association with plants $[9,10]$. Based on the effective strain of agrobacteria the microbial preparation Diazofit was developed, recommended for rice and wheat inoculation [9]. Positive action of Diazofit on these crops was shown in many field and production experiments in different soil climatic conditions [11]. Use of certain strains of Rhizobium radiobacter promotes higher molecular nitrogen fixation activity in the root zone of winter rye plants and culture productivity [3]. However, experiments with spring barley failed to show active development of agrobacteria in plants rhizosphere, while application of certain Rhizobium radiobacter strains has not influenced the process of associative nitrogen fixation [12].

Thus, the positive result of analytical selection of diazotrophic bacteria and creation of effective plant-microbe associations is possible only with thorough selection of micro-and macroorganisms combined with the systematic study of all aspects of nitrogen fixing bacteria interactions with plants.

Summarizing the results obtained, the following conclusions can be drawn. From the root zone of spring wheat plants grown on leached black soils 310 diazotrophs cultures possessing high ability to fix atmospheric nitrogen were isolated 
and identified as the representatives of Azotobacter, Azospirillum, Bacillus, Flavobacterium, Enterobacter and Pseudomonas genera. It was established that diazotrophs isolated from the root zone of spring wheat were able to form associative system with plants, with Azospirillum - spring wheat plant association demonstrating the largest nitrogenase activity. 